\title{
An extremization principle for the entropy of rotating BPS black holes in $\mathrm{AdS}_{5}$
}

\author{
Seyed Morteza Hosseini, ${ }^{a, b}$ Kiril Hristov $^{c}$ and Alberto Zaffaroni ${ }^{a, b}$ \\ ${ }^{a}$ Dipartimento di Fisica, Università di Milano-Bicocca, \\ I-20126 Milano, Italy \\ ${ }^{b}$ INFN, sezione di Milano-Bicocca, \\ I-20126 Milano, Italy \\ ${ }^{c}$ Institute for Nuclear Research and Nuclear Energy, Bulgarian Academy of Sciences, \\ Tsarigradsko Chaussee 72, 1784 Sofia, Bulgaria \\ E-mail: morteza.hosseini@mib.infn.it, khristov@inrne.bas.bg, \\ alberto.zaffaroni@mib.infn.it
}

ABSTRACT: We show that the Bekenstein-Hawking entropy of a class of BPS electrically charged rotating black holes in $\mathrm{AdS}_{5} \times S^{5}$ can be obtained by a simple extremization principle. We expect that this extremization corresponds to the attractor mechanism for BPS rotating black holes in five-dimensional gauged supergravity, which is still unknown. The expression to be extremized has a suggestive resemblance to anomaly polynomials and the supersymmetric Casimir energy recently studied for $\mathcal{N}=4$ super Yang-Mills.

KEYwords: AdS-CFT Correspondence, Black Holes in String Theory, Supergravity Models

ARXIV EPRINT: 1705.05383 


\section{Contents}

1 Introduction $\quad 1$

2 Supersymmetric $\mathbf{A d S}_{5}$ black holes in $\mathrm{U}(1)^{3}$ gauged supergravity 3

2.1 The asymptotic $\mathrm{AdS}_{5}$ vacuum 5

2.2 Properties of the solution 5

$\begin{array}{lll}3 & \text { An extremization principle for the entropy } & 6\end{array}$

4 Dimensional reduction in the limiting case: $J_{\phi}=J_{\psi} \quad 8$

4.1 The near-horizon geometry 8

4.2 Dimensional reduction on the Hopf fibres of squashed $S^{3} \quad 10$

$\begin{array}{ll}4.3 & \text { Attractor mechanism in four dimensions } \\ & 12\end{array}$

$\begin{array}{ll}4.4 \text { Comparison with five-dimensional extremization } & 13\end{array}$

$\begin{array}{lll}5 & \text { Discussion and future directions } & 13\end{array}$

$\begin{array}{ll}\text { A Five-dimensional } \mathcal{N}=2 \text { gauged supergravity } & 16\end{array}$

$\begin{array}{ll}\text { B Four-dimensional } \mathcal{N}=2 \text { gauged supergravity } & 17\end{array}$

$\begin{array}{ll}\text { C Generalities about the supersymmetric Casimir energy } & 19\end{array}$

\section{Introduction}

The derivation of the entropy of BPS electrically charged rotating black holes in $\mathrm{AdS}_{5} \times$ $S^{5}[1-5]$ in terms of states of the dual $\mathcal{N}=4 \mathrm{SU}(N)$ super Yang-Mills (SYM) theory is still an open problem. Various attempts have been made in this direction [6-8] but none was really successful. One could consider the superconformal index $[6,9]$ since it counts states preserving the same supersymmetries of the black holes and it depends on a number of fugacities equal to the number of conserved charges of the black holes. However, due to a large cancellation between bosonic and fermionic states, the index is a quantity of order one for generic values of the fugacities while the entropy scales like $N^{2}$ [6]. We also know that the supersymmetric partition function on $S^{3} \times S^{1}$ is equal to the superconformal index only up to a multiplicative factor $e^{-\beta E_{\mathrm{SUSY}}}$, where the supersymmetric Casimir energy $E_{\mathrm{SUSY}}$ is of order $N^{2}$ [10-16]. However, it is not clear what the average energy of the vacuum should have to do with the entropy, which is the degeneracy of ground states. The analogous problem for static, asymptotically $\mathrm{AdS}_{4}$ dyonic black holes was recently solved in [17, 18]. It was shown in $[17,18]$ that the topologically twisted index for three-dimensional gauge 
theories $[19]^{1}$ has no large cancellation between bosons and fermions at large $N$, it scales like $N^{3 / 2}[17,24,25]$ (see also [26]) and correctly reproduces the entropy of a class of BPS black holes in $\mathrm{AdS}_{4} \times S^{7}$. More precisely, the topologically twisted index $Z_{\text {twisted }}$ is a function of magnetic charges $p_{i}$ and fugacities $\Delta_{i}$ for the global symmetries of the theory. The entropy of the black holes with electric charges $q_{i}$ is then obtained as a Legendre transform of $\log Z_{\text {twisted }}$ :

$$
S\left(q_{i}, p_{i}\right)=\log Z_{\text {twisted }}\left(p_{i}, \Delta_{i}\right)-\left.i \sum_{i} q_{i} \Delta_{i}\right|_{\bar{\Delta}_{i}}
$$

where $\bar{\Delta}_{i}$ is the extremum of $\mathcal{I}\left(\Delta_{i}\right)=\log Z_{\text {twisted }}\left(p_{i}, \Delta_{i}\right)-i q_{i} \Delta_{i}$. This procedure has been called $\mathcal{I}$-extremization in $[17,18]$ and shown to correspond to the attractor mechanism in gauged supergravity [27-33].

It is natural to ask what would be the analogous of this construction in five dimensions. In this paper, we humbly look attentively at the gravity side of the story and try to understand what kind of extremization can reproduce the entropy of the supersymmetric rotating black holes. Unfortunately, the details of the attractor mechanism for rotating black holes in five-dimensional gauged supergravity are not known but we can nevertheless find an extremization principle for the entropy. The final result is quite surprising and intriguing.

We consider the class of supersymmetric rotating black holes found and studied in $[1-5]$. They are asymptotic to $\mathrm{AdS}_{5} \times S^{5}$ and depend on three electric charges $Q_{I}(I=1,2,3)$, associated with rotations in $S^{5}$, and two angular momenta $J_{\phi}, J_{\psi}$ in $\mathrm{AdS}_{5}$. Supersymmetry actually requires a constraint among the charges and only four of them are independent. We show that the Bekenstein-Hawking entropy of the black holes can be obtained as the Legendre transform of the quantity ${ }^{2}$

$$
E=-i \pi N^{2} \frac{\Delta_{1} \Delta_{2} \Delta_{3}}{\omega_{1} \omega_{2}}
$$

where $\Delta_{I}$ are chemical potentials conjugated to the electric charges $Q_{I}$ and $\omega_{1,2}$ chemical potentials conjugated to the angular momenta $J_{\phi}, J_{\psi}$. The constraint among charges is reflected in the following constraint among chemical potentials,

$$
\Delta_{1}+\Delta_{2}+\Delta_{3}+\omega_{1}+\omega_{2}=1 .
$$

To further motivate the result (1.2) we shall consider the case of equal angular momenta $J_{\psi}=J_{\phi}$. In this limit, the black hole has an enhanced $\mathrm{SU}(2) \times \mathrm{U}(1)$ isometry and it can be reduced along the $\mathrm{U}(1)$ to a static dyonic black hole in four dimensions. We show that, upon dimensional reduction, the extremization problem based on (1.2) coincides with the attractor mechanism in four dimensions, which is well understood for static BPS black holes [27-33].

\footnotetext{
${ }^{1}$ For further developments see [20-23].

${ }^{2}$ Notice that one can write the very same entropy as the result of a different extremization in the context of the Sen's entropy functional [34, 35]. The two extremizations are over different quantities and use different charges.
} 
It is curious to observe that the expression (1.2) is formally identical to the supersymmetric Casimir energy for $\mathcal{N}=4 \mathrm{SYM}$, as derived, for example, in [13] and reviewed in appendix C. It appears in the relation $Z_{\mathcal{N}=4}=e^{-E} I$ between the partition function $Z_{\mathcal{N}=4}$ on $S^{3} \times S^{1}$ and the superconformal index $I$. Both the partition function and the superconformal index are functions of a set of chemical potentials $\Delta_{I}(I=1,2,3)$ and $\omega_{i}$ $(i=1,2)$ associated with the R-symmetry generators $\mathrm{U}(1)^{3} \in \mathrm{SO}(6)$ and the two angular momenta $\mathrm{U}(1)^{2} \in \mathrm{SO}(4)$, respectively. Since the symmetries that appear in the game must commute with the preserved supercharge, the index and the partition function are actually functions of only four independent chemical potentials, precisely as our quantity $E$. The constraint among chemical potentials is usually imposed as $\sum_{I=1}^{3} \Delta_{I}+\sum_{i=1}^{2} \omega_{i}=0$. Since chemical potentials in our notations are periodic of period 1 , our constraint (1.3) reflects a different choice for the angular ambiguities. We comment about the interpretation of this result in the discussion section, leaving the proper understanding to future work.

The paper is organized as follows. In section 2 we review the basic features of the BPS rotating black holes of interest. In section 3, we show that the Bekenstein-Hawking entropy of the black hole can be obtained as the Legendre transform of the quantity (1.2). In section 4, we perform the dimensional reduction of the black holes with equal angular momenta down to four dimensions and we prove that the extremization of (1.2) is equivalent to the attractor mechanism for four-dimensional static BPS black holes in gauged supergravity. We conclude in section 5 with discussions and future directions. The appendices contain the relevant information and conventions about gauged supergravity in five and four dimensions and the supersymmetric Casimir energy.

\section{Supersymmetric $\mathrm{AdS}_{5}$ black holes in $\mathrm{U}(1)^{3}$ gauged supergravity}

In this section we will briefly review a class of supersymmetric, asymptotically AdS, black holes of $D=5 \mathrm{U}(1)^{3}$ gauged supergravity [1-5]. They can be embedded in type IIB supergravity as an asymptotically $\operatorname{AdS}_{5} \times S^{5}$ solution which is exactly the decoupling limit of the rotating D3-brane [36]. Further details about the supergravity model can be found in [36] and appendix A. When lifted to type IIB supergravity they preserve only two real supercharges [37]. They are characterized by their mass, three electric charges and two angular momenta with a constraint, and are holographically dual to $1 / 16$ BPS states of $\mathcal{N}=4 \mathrm{SU}(N)$ SYM theory on $S^{3} \times \mathbb{R}$ at large $N$.

We shall primarily be interested in the so-called $\mathcal{N}=2$ gauged STU model, that arises upon compactification of type IIB supergravity on $S^{5}$. In the notations of appendix A, the only nonvanishing triple intersection numbers are $C_{123}=1$ (and cyclic permutations). The bosonic sector of the theory comprises three gauge fields which correspond to the Cartan subalgebra of the $\mathrm{SO}(6)$ isometry of $S^{5}$, the metric, and three real scalar fields subject to the constraint

$$
L^{1} L^{2} L^{3}=1
$$

They take vacuum values $\bar{L}^{I}=1$. The five-dimensional black hole metric can be writ- 
ten as $[5]$

$$
d s^{2}=-\left(H_{1} H_{2} H_{3}\right)^{-2 / 3}\left(d t+\omega_{\psi} d \psi+\omega_{\phi} d \phi\right)^{2}+\left(H_{1} H_{2} H_{3}\right)^{1 / 3} h_{m n} d x^{m} d x^{n},
$$

where

$$
\begin{aligned}
H_{I}= & 1+\frac{\sqrt{\Xi_{a} \Xi_{b}}\left(1+g^{2} \mu_{I}\right)-\Xi_{a} \cos ^{2} \theta-\Xi_{b} \sin ^{2} \theta}{g^{2} r^{2}}, \\
h_{m n} d x^{m} d x^{n}= & r^{2}\left\{\frac{d r^{2}}{\Delta_{r}}+\frac{d \theta^{2}}{\Delta_{\theta}}+\frac{\cos ^{2} \theta}{\Xi_{b}^{2}}\left[\Xi_{b}+\cos ^{2} \theta\left(\rho^{2} g^{2}+2(1+b g)(a+b) g\right)\right] d \psi^{2}\right. \\
& +\frac{\sin ^{2} \theta}{\Xi_{a}^{2}}\left[\Xi_{a}+\sin ^{2} \theta\left(\rho^{2} g^{2}+2(1+a g)(a+b) g\right)\right] d \phi^{2} \\
& \left.+\frac{2 \sin ^{2} \theta \cos ^{2} \theta}{\Xi_{a} \Xi_{b}}\left[\rho^{2} g^{2}+2(a+b) g+(a+b)^{2} g^{2}\right] d \psi d \phi\right\} \\
\Delta_{r}= & r^{2}\left[g^{2} r^{2}+(1+a g+b g)^{2}\right], \quad \Delta_{\theta}=\Xi_{a} \cos ^{2} \theta+\Xi_{b} \sin ^{2} \theta, \\
\Xi_{a}= & 1-a^{2} g^{2}, \quad \Xi_{b}=1-b^{2} g^{2}, \quad \rho^{2}=r^{2}+a^{2} \cos ^{2} \theta+b^{2} \sin ^{2} \theta \\
\omega_{\psi}= & -\frac{g \cos ^{2} \theta}{r^{2} \Xi_{b}}\left[\rho^{4}+\left(2 r_{m}^{2}+b^{2}\right) \rho^{2}+\frac{1}{2}\left(\beta_{2}-a^{2} b^{2}+g^{-2}\left(a^{2}-b^{2}\right)\right)\right], \\
\omega_{\phi}= & -\frac{g \sin ^{2} \theta}{r^{2} \Xi_{a}}\left[\rho^{4}+\left(2 r_{m}^{2}+a^{2}\right) \rho^{2}+\frac{1}{2}\left(\beta_{2}-a^{2} b^{2}-g^{-2}\left(a^{2}-b^{2}\right)\right)\right],
\end{aligned}
$$

and

$$
\begin{aligned}
& r_{m}^{2}=g^{-1}(a+b)+a b, \\
& \beta_{2}=\Xi_{a} \Xi_{b}\left(\mu_{1} \mu_{2}+\mu_{1} \mu_{3}+\mu_{2} \mu_{3}\right)-\frac{2 \sqrt{\Xi_{a} \Xi_{b}}\left(1-\sqrt{\Xi_{a} \Xi_{b}}\right)}{g^{2}}\left(\mu_{1}+\mu_{2}+\mu_{3}\right)+\frac{3\left(1-\sqrt{\Xi_{a} \Xi_{b}}\right)^{2}}{g^{4}} .
\end{aligned}
$$

The gauge coupling constant $g$ is fixed in terms of the $\mathrm{AdS}_{5}$ radius of curvature, $g=1 / \ell$. The coordinates are $(t, r, \theta, \phi, \psi)$ where $r>0$ corresponds to the exterior of the black hole, $0 \leq \theta \leq \pi / 2$ and $0 \leq \phi, \psi \leq 2 \pi$. The scalars read

$$
L^{I}=\frac{\left(H_{1} H_{2} H_{3}\right)^{1 / 3}}{H_{I}},
$$

while the gauge potentials are given by

$$
A^{I}=H_{I}^{-1}\left(d t+\omega_{\psi} d \psi+\omega_{\phi} d \phi\right)+U_{\psi}^{I} d \psi+U_{\phi}^{I} d \phi
$$

where

$$
\begin{aligned}
& U_{\psi}^{I}=\frac{g \cos ^{2} \theta}{\Xi_{b}}\left[\rho^{2}+2 r_{m}^{2}+b^{2}-\sqrt{\Xi_{a} \Xi_{b}} \mu_{I}+g^{-2}\left(1-\sqrt{\Xi_{a} \Xi_{b}}\right)\right], \\
& U_{\phi}^{I}=\frac{g \sin ^{2} \theta}{\Xi_{a}}\left[\rho^{2}+2 r_{m}^{2}+a^{2}-\sqrt{\Xi_{a} \Xi_{b}} \mu_{I}+g^{-2}\left(1-\sqrt{\Xi_{a} \Xi_{b}}\right)\right] .
\end{aligned}
$$


The black hole is labeled by five parameters: $\mu_{1,2,3}, a, b$ where $g^{-1}>a, b \geq 0$. Only four parameters are independent due to the constraint

$$
\mu_{1}+\mu_{2}+\mu_{3}=\frac{1}{\sqrt{\Xi_{a} \Xi_{b}}}\left[2 r_{m}^{2}+3 g^{-2}\left(1-\sqrt{\Xi_{a} \Xi_{b}}\right)\right]
$$

Furthermore, regularity of the scalars for $r \geq 0$ entails that

$$
g^{2} \mu_{I}>\sqrt{\frac{\Xi_{b}}{\Xi_{a}}}-1 \geq 0
$$

when $a \geq b$. If $a<b$ then the same expression remains valid with $a$ and $b$ interchanged.

\subsection{The asymptotic $\operatorname{AdS}_{5}$ vacuum}

This solution is expressed in the co-rotating frame. The change of coordinates $t=\bar{t}$, $\phi=\bar{\phi}-g \bar{t}, \psi=\bar{\psi}-g \bar{t}$, and $y^{2}=r^{2}+2 r_{m}^{2} / 3$ transforms the metric to a static frame at infinity. In order to bring the metric into a manifestly asymptotically $\mathrm{AdS}_{5}$ spacetime (in the global sense) as $y \rightarrow \infty$ we make the following change of coordinates [5]

$$
\Xi_{a} Y^{2} \sin ^{2} \Theta=\left(y^{2}+a^{2}\right) \sin ^{2} \theta, \quad \Xi_{b} Y^{2} \cos ^{2} \Theta=\left(y^{2}+b^{2}\right) \cos ^{2} \theta .
$$

One gets the line element

$$
d s^{2} \simeq-g^{2} Y^{2} d \bar{t}^{2}+\frac{d Y^{2}}{g^{2} Y^{2}}+Y^{2}\left(d \Theta^{2}+\sin ^{2} \Theta d \bar{\phi}^{2}+\cos ^{2} \Theta d \bar{\psi}^{2}\right) .
$$

The black hole has the Einstein universe $\mathbb{R} \times S^{3}$ as its conformal boundary. In the asymptotically static coordinates, the supersymmetric Killing vector field reads

$$
V=\frac{\partial}{\partial \bar{t}}+g \frac{\partial}{\partial \bar{\phi}}+g \frac{\partial}{\partial \bar{\psi}}
$$

which is timelike everywhere outside the black hole and is null on the conformal boundary.

\subsection{Properties of the solution}

It is convenient to define the following polynomials,

$$
\gamma_{1}=\mu_{1}+\mu_{2}+\mu_{3}, \quad \gamma_{2}=\mu_{1} \mu_{2}+\mu_{1} \mu_{3}+\mu_{2} \mu_{3}, \quad \gamma_{3}=\mu_{1} \mu_{2} \mu_{3} .
$$

The black hole carries three $\mathrm{U}(1)^{3} \subset \mathrm{SO}(6)$ electric charges in $S^{5}$ which are given by

$$
Q_{I}=\frac{\pi}{4 G_{\mathrm{N}}^{(5)}}\left[\mu_{I}+\frac{g^{2}}{2}\left(\gamma_{2}-\frac{2 \gamma_{3}}{\mu_{I}}\right)\right], \quad \text { for } \quad I=1,2,3,
$$

and two $\mathrm{U}(1)^{2} \subset \mathrm{SO}(4)$ angular momenta in $\mathrm{AdS}_{5}$ that read

$$
\begin{aligned}
& J_{\psi}=\frac{\pi}{4 G_{\mathrm{N}}^{(5)}}\left[\frac{g \gamma_{2}}{2}+g^{3} \gamma_{3}+g^{-3}\left(\sqrt{\frac{\Xi_{a}}{\Xi_{b}}}-1\right) \mathcal{J}\right], \\
& J_{\phi}=\frac{\pi}{4 G_{\mathrm{N}}^{(5)}}\left[\frac{g \gamma_{2}}{2}+g^{3} \gamma_{3}+g^{-3}\left(\sqrt{\frac{\Xi_{b}}{\Xi_{a}}}-1\right) \mathcal{J}\right] .
\end{aligned}
$$


Here, $G_{\mathrm{N}}^{(5)}$ is the five-dimensional Newton constant and we defined

$$
\mathcal{J} \equiv \prod_{I=1}^{3}\left(1+g^{2} \mu_{I}\right)
$$

The mass of the black holes is determined by the BPS condition

$$
M=g\left|J_{\phi}\right|+g\left|J_{\psi}\right|+\left|Q_{1}\right|+\left|Q_{2}\right|+\left|Q_{3}\right|
$$

which yields

$$
M=\frac{\pi}{4 G_{\mathrm{N}}^{(5)}}\left[\gamma_{1}+\frac{3 g^{2} \gamma_{2}}{2}+2 g^{4} \gamma_{3}+\frac{\left(\sqrt{\Xi_{a}}-\sqrt{\Xi_{b}}\right)^{2}}{g^{2} \sqrt{\Xi_{a} \Xi_{b}}} \mathcal{J}\right]
$$

The solution has a regular event horizon at $r_{\mathrm{h}}=0$ only for nonzero angular momenta in $\mathrm{AdS}_{5}$. The angular velocities of the horizon, measured with respect to the azimuthal coordinates $\psi$ and $\phi$ of the asymptotically static frame at infinity, are

$$
\Omega_{\psi}=\Omega_{\phi}=g
$$

The near-horizon geometry is a fibration of $\mathrm{AdS}_{2}$ over a non-homogeneously squashed $S^{3}[38]$ with area

$$
\text { Area }=2 \pi^{2} \sqrt{\gamma_{3}\left(1+g^{2} \gamma_{1}\right)-\frac{g^{2} \gamma_{2}^{2}}{4}-\frac{\left(\sqrt{\Xi_{a}}-\sqrt{\Xi_{b}}\right)^{2}}{g^{6} \sqrt{\Xi_{a} \Xi_{b}}} \mathcal{J}} .
$$

Positivity of the expression within the square root ensures the absence of closed causal curves near $r=0$. The Bekenstein-Hawking entropy of the black hole is proportional to its horizon area and can be compactly written in terms of the physical charges as [39]

$$
S_{\mathrm{BH}}=\frac{\text { Area }}{4 G_{\mathrm{N}}^{(5)}}=\frac{2 \pi}{g} \sqrt{Q_{1} Q_{2}+Q_{2} Q_{3}+Q_{1} Q_{3}-\frac{\pi}{4 G_{\mathrm{N}}^{(5)} g}\left(J_{\phi}+J_{\psi}\right)} .
$$

Finally, let

$$
\mathcal{X}_{I}=\left(1+g^{2} \mu_{I}\right) \sqrt{\Xi_{a} \Xi_{b}}-\Delta_{\theta} .
$$

The values of the scalar fields at the horizon read

$$
L^{I}\left(r_{\mathrm{h}}\right)=\frac{\left(\mathcal{X}_{1} \mathcal{X}_{2} \mathcal{X}_{3}\right)^{1 / 3}}{\mathcal{X}_{I}}
$$

In the next section we will obtain the Bekenstein-Hawking entropy (2.24) of the BPS black hole from an extremization principle.

\section{An extremization principle for the entropy}

We shall now extremize the quantity (1.2), and show that the extremum precisely reproduces the entropy of the multi-charge BPS black hole discussed in the previous section. 
Let us first introduce some notation that facilitates the comparison with supergravity:

$$
X^{I} \equiv \Delta_{I}, \quad X_{ \pm}^{0} \equiv \omega_{1} \pm \omega_{2},
$$

where $I=1,2,3$. We shall also use $J^{ \pm} \equiv J_{\phi} \pm J_{\psi}$,

$$
\begin{aligned}
& J^{+}=\frac{\pi}{4 G_{\mathrm{N}}^{(5)}}\left[g \gamma_{2}+2 g^{3} \gamma_{3}+\frac{\left(\sqrt{\Xi_{a}}-\sqrt{\Xi_{b}}\right)^{2}}{g^{3} \sqrt{\Xi_{a} \Xi_{b}}} \mathcal{J}\right], \\
& J^{-}=\frac{\pi}{4 G_{\mathrm{N}}^{(5)}} \frac{\Xi_{b}-\Xi_{a}}{g^{3} \sqrt{\Xi_{a} \Xi_{b}}} \mathcal{J} .
\end{aligned}
$$

Thus, we can rewrite the quantity (1.2) as

$$
E=-\frac{2 \pi^{2} i}{g^{3} G_{\mathrm{N}}^{(5)}} \frac{X^{1} X^{2} X^{3}}{\left(X_{+}^{0}\right)^{2}-\left(X_{-}^{0}\right)^{2}}
$$

where we used the standard relation between gravitational and QFT parameters in the large $N$ limit,

$$
\frac{\pi}{2 g^{3} G_{\mathrm{N}}^{(5)}}=N^{2}
$$

In the following we set the unit of the $\mathrm{AdS}_{5}$ curvature $g=1$. The entropy of the BPS black hole, at leading order, can be obtained by extremizing the quantity ${ }^{3}$

$$
\mathcal{S}=-E\left(X_{ \pm}^{0}, X^{I}\right)+2 \pi i \sum_{I=1}^{3} Q_{I} X^{I}-\pi i\left(J^{+} X_{+}^{0}+J^{-} X_{-}^{0}\right)
$$

with respect to $X^{I}, X_{ \pm}^{0}$ and subject to the constraint (1.3),

$$
X_{+}^{0}+\sum_{I=1}^{3} X^{I}=1
$$

At this stage, we find it more convenient to work in the basis $z^{\alpha}(\alpha=0,1,2,3)$ which is related to $\left(X_{ \pm}^{0}, X^{I}\right)$ by

$$
\begin{array}{rlrl}
X_{-}^{0} & =\frac{z^{0}}{1+z^{1}+z^{2}+z^{3}}, & X_{+}^{0}=\frac{1}{1+z^{1}+z^{2}+z^{3}}, \\
X^{1,2,3} & =\frac{z^{1,2,3}}{1+z^{1}+z^{2}+z^{3}} .
\end{array}
$$

Hence, in terms of the variables $z^{\alpha}$ the extremization equations can be written as

$$
\begin{gathered}
{\left[\left(z^{0}\right)^{2}-1\right]\left\{\left[\left(z^{0}\right)^{2}-1\right] c^{i}+\frac{z^{1} z^{2} z^{3}}{z^{i}}\right\}-2 z^{1} z^{2} z^{3}=0, \quad \text { for } \quad i=1,2,3,} \\
c^{0}\left[\left(z^{0}\right)^{2}-1\right]^{2}-2 z^{0} z^{1} z^{2} z^{3}=0
\end{gathered}
$$

${ }^{3}$ This is not the only possible choice of signs. There are various sign ambiguities in the superconformal index literature as well as in the black hole one that should be fixed in a proper comparison between gravity and field theory. 
where we defined the constants

$$
c^{0}=\frac{\mathcal{J}\left(\Xi_{b}-\Xi_{a}\right)}{8 \sqrt{\Xi_{a} \Xi_{b}}}, \quad \quad c^{i}=\frac{\mathcal{J}}{4}\left(\frac{1}{1+\mu_{i}}-\frac{\Xi_{b}+\Xi_{a}}{2 \sqrt{\Xi_{a} \Xi_{b}}}\right) .
$$

With an explicit computation one can check that the value of $\mathcal{S}\left(z^{\alpha}\right)$ at the critical point precisely reproduces the entropy of the black hole,

$$
\left.\mathcal{S}\right|_{\text {crit }}\left(J^{ \pm}, Q_{I}\right)=S_{\mathrm{BH}}\left(J^{ \pm}, Q_{I}\right)
$$

It is remarkable that the solution to the extremization equations (3.8) is complex; however, at the saddle-point it becomes a real function of the black hole charges. We conclude that the extremization of the quantity (1.2) yields exactly the Bekenstein-Hawking entropy of the $1 / 16$ BPS black holes in $\mathrm{AdS}_{5} \times S^{5}$.

So far the discussion was completely general. In the next section, we will analyze the case $J_{\phi}=J_{\psi}$, for which the solution to the extremization equations takes a remarkably simple form.

\section{Dimensional reduction in the limiting case: $J_{\phi}=J_{\psi}$}

We gain some important insight by considering the dimensional reduction of the fivedimensional BPS black holes when the two angular momenta are equal. The black hole metric on the squashed sphere then has an enhanced isometry $\mathrm{SU}(2) \times \mathrm{U}(1) \subset \mathrm{SO}(4)$. If we choose the appropriate Hopf coordinates we can dimensionally reduce the solution along the $\mathrm{U}(1)$ down to four-dimensional gauged supergravity. As discussed in [40], it turns out that such a dimensional reduction makes sense not only for asymptotically flat solutions where first discovered in [41-44] but also for the asymptotically AdS solutions in the gauged supergravity considered here. A crucial difference is that the lower-dimensional vacuum will no longer be maximally symmetric but will instead be of the hyperscaling-violating Lifshitz (hvLif) type [40].

The reason for looking at the limit $J_{\phi}=J_{\psi}$ is simple: due to the $\mathrm{SU}(2)$ symmetry the lower-dimensional solution is guaranteed to be static and the horizon metric is a direct product $\mathrm{AdS}_{2} \times S^{2}$ geometry, as will be shown in due course. Since the attractor mechanism for static BPS black holes in four-dimensional $\mathcal{N}=2$ gauged supergravity has been completely understood [28-30] we can fit the reduced solution in this framework.

\subsection{The near-horizon geometry}

We begin by taking the near-horizon limit, $r \rightarrow 0$, of the BPS black hole solution presented in section 2. We set $a=b$, corresponding to the equal angular momenta $\left(J_{\psi}=J_{\phi}\right)$, and adopt the notation $\Xi_{a}=\Xi_{b} \equiv \Xi$.

Let us first introduce the following coordinates,

$$
\psi \equiv \frac{1}{2}(\chi+\varphi), \quad \phi \equiv \frac{1}{2}(\chi-\varphi), \quad \theta \equiv \frac{1}{2} \vartheta,
$$


where $\vartheta, \varphi, \chi$ are the Euler angles of $S^{3}$ with $0 \leq \vartheta \leq \pi, 0 \leq \varphi<2 \pi, 0 \leq \chi<4 \pi$. The near-horizon geometry then reads

$$
\begin{aligned}
d s^{2} & =R_{\mathrm{AdS}_{2}}^{2} d s_{\mathrm{AdS}_{2}}^{2}+\gamma_{3}^{1 / 3} d s_{\mathcal{M}_{3}}^{2}, \\
L^{I} & =\frac{\gamma_{3}^{1 / 3}}{\mu_{I}}, \quad A^{I}=\frac{\gamma_{3}^{1 / 3}}{\mu_{I}} R_{\mathrm{AdS}_{2}} \tilde{r} d \tilde{t}+g\left(\gamma_{1}-\mu_{I}-\frac{\gamma_{2}}{2 \mu_{I}}\right) d \gamma,
\end{aligned}
$$

where we defined

$$
\begin{aligned}
d s_{\mathrm{AdS}_{2}}^{2} & =-\tilde{r}^{2} d \tilde{t}^{2}+\frac{d \tilde{r}^{2}}{\tilde{r}^{2}}, \quad R_{\mathrm{AdS}_{2}}^{2}=\frac{\gamma_{3}^{1 / 3}}{4\left(1+g^{2} \gamma_{1}\right)}, \\
\tilde{r} & =\frac{r^{2}}{4 R_{\mathrm{AdS}_{2}}^{2}}, \quad \tilde{t}=\frac{2}{\Xi \sqrt{\gamma_{3}^{1 / 3}\left(1+g^{2} \gamma_{1}\right)}} t, \\
d s_{\mathcal{M}_{3}}^{2} & =d s_{S^{3}}^{2}-\left[\Gamma^{2} \gamma_{3}^{1 / 3}-\frac{a g(4+5 a g)}{\Xi}\right] d \gamma^{2}+2 R_{\mathrm{AdS}_{2}} \Gamma \tilde{r} d \tilde{t} d \gamma \\
\Gamma & =\frac{g\left(3 a^{4}+4 a^{2} r_{m}^{2}+\beta_{2}\right)}{2 \Xi^{2} \gamma_{3}^{2 / 3}},
\end{aligned}
$$

and

$$
d s_{S^{3}}^{2}=\frac{1}{4}\left(d \vartheta^{2}+d \varphi^{2}+d \chi^{2}+2 \cos \vartheta d \varphi d \chi\right)=\frac{1}{4} \sum_{i=1}^{3} \sigma_{i}, \quad d \gamma=\frac{\sigma_{3}}{2} .
$$

Here, $\sigma_{i}(i=1,2,3)$ are the right-invariant $\mathrm{SU}(2)$ one-forms,

$$
\begin{aligned}
& \sigma_{1}=-\sin \chi d \vartheta+\cos \chi \sin \vartheta d \varphi \\
& \sigma_{2}=\cos \chi d \vartheta+\sin \chi \sin \vartheta d \varphi \\
& \sigma_{3}=d \chi+\cos \vartheta d \varphi
\end{aligned}
$$

Notice that

$$
d s_{S^{2}}^{2}=\sigma_{1}^{2}+\sigma_{2}^{2}=d \vartheta^{2}+\sin ^{2} \vartheta d \varphi^{2} .
$$

Due to the constraint (2.11) we can simplify

$$
\frac{a(4+5 a g)}{\Xi}=g \gamma_{1}, \quad \quad \Gamma=\frac{g \gamma_{2}}{2 \gamma_{3}^{2 / 3}} .
$$

Upon a further rescaling of the time coordinate

$$
\tilde{t}=-\frac{1}{2} \sqrt{4-\frac{g^{2} \gamma_{2}^{2}}{\gamma_{3}\left(1+g^{2} \gamma_{1}\right)}} \tau,
$$

the near-horizon metric with squashed $\mathrm{AdS}_{2} \times_{w} S^{3}$ geometry and the gauge fields can be brought to the form:

$$
\begin{aligned}
d s_{(5)}^{2} & =R_{\mathrm{AdS}_{2}}^{2} d s_{\mathrm{AdS}_{2}}^{2}+\frac{R_{S^{2}}^{2}}{4}\left[d s_{S^{2}}^{2}+v\left(\sigma_{3}-\alpha \tilde{r} d \tau\right)^{2}\right], \\
L^{I} & =\frac{\gamma_{3}^{1 / 3}}{\mu_{I}}, \quad A_{(5)}^{I}=e^{I} \tilde{r} d \tau-f^{I} \sigma_{3} .
\end{aligned}
$$


Here, we defined the constants

$$
\begin{aligned}
\alpha & =\frac{g \gamma_{2}}{2\left(1+g^{2} \gamma_{1}\right) \sqrt{\gamma_{3} v}}, & R_{S^{2}}^{2} & =\gamma_{3}^{1 / 3}, \\
e^{I} & =-\frac{\sqrt{\gamma_{3} v}}{2 \mu_{I}\left(1+g^{2} \gamma_{1}\right)}, & f^{I} & =\frac{g}{4}\left(\mu_{I}-\gamma_{1}\right)+\frac{g \gamma_{3}}{4 \mu_{I}^{2}},
\end{aligned}
$$

and

$$
v=1+g^{2} \gamma_{1}-\frac{g^{2} \gamma_{2}^{2}}{4 \gamma_{3}} .
$$

Note that we added the subscript (5) in order to emphasize that these are five-dimensional quantities which will next be related to a solution in four dimensions via dimensional reduction along the $\chi$ direction.

\subsection{Dimensional reduction on the Hopf fibres of squashed $S^{3}$}

In five-dimensional supergravity theories, including $n_{\mathrm{V}}$ abelian gauge fields $A_{(5)}^{I}$ and real scalar fields $L^{I}\left(I=1, \ldots, n_{\mathrm{V}}\right)$ coupled to gravity, the rules for reducing the bosonic fields are the following $[43,45-48]$ :

$$
\begin{array}{rlrl}
d s_{(5)}^{2} & =e^{2 \phi} d s_{(4)}^{2}+e^{-4 \phi}\left(d x^{5}-A_{(4)}^{0}\right)^{2}, & & d x^{5}=d \chi, \\
A_{(5)}^{I} & =A_{(4)}^{I}+\operatorname{Re} z^{I}\left(d x^{5}-A_{(4)}^{0}\right), & & \\
L^{I} & =e^{2 \phi} \mathbb{I m} z^{I}, & e^{-6 \phi}=\frac{1}{6} C_{I}
\end{array}
$$

where $d s_{(4)}^{2}$ denotes the four-dimensional line element, the $A_{(4)}^{\Lambda}(\Lambda=0, I)$ are the fourdimensional abelian gauge fields and $z^{I}$ are the complex scalar fields in four dimensions. Our conventions for $\mathcal{N}=2$ gauged supergravity in four dimensions are presented in appendix B. The four-dimensional theory has $n_{\mathrm{V}}$ abelian vector multiplets, parameterizing a special Kähler manifold $\mathcal{M}$ with metric $g_{i \bar{j}}$, in addition to the gravity multiplet (thus a total of $n_{\mathrm{V}}+1$ gauge fields and $n_{\mathrm{V}}$ complex scalars). The scalar manifold is defined by the prepotential $\mathcal{F}\left(X^{\Lambda}\right)$, which is a homogeneous holomorphic function of sections $X^{\Lambda}$,

$$
\mathcal{F}\left(X^{\Lambda}\right)=-\frac{1}{6} \frac{C_{I J K} X^{I} X^{J} X^{K}}{X^{0}}=-\frac{X^{1} X^{2} X^{3}}{X^{0}} .
$$

In the second equality we employed the five-dimensional supergravity data for the STU model from section 2 . In $\mathcal{N}=2$ gauged supergravity in four dimensions the $\mathrm{U}(1)_{R}$ symmetry, rotating the gravitini, is gauged by a linear combination of the (now four) abelian gauge fields. The coefficients are called Fayet-Iliopoulos (FI) parameters $g_{\Lambda}$ and three of them can be directly read off the five-dimensional theory: $g_{1}=g_{2}=g_{3}=1 .{ }^{4}$ The last coefficient, $g_{0}$, measuring how the Kaluza-Klein gauge potential $A_{(4)}^{0}$ enters the R-symmetry, can be left arbitrary for the moment. This can be achieved by a Scherk-Schwarz reduction when allowing a particular reduction ansatz for the gravitino as explained in [45, 48-51]. The

\footnotetext{
${ }^{4}$ In consistent models one can always apply an electric-magnetic duality transformation so that the corresponding gauging becomes purely electric, i.e., $g^{\Lambda}=0$.
} 
prepotential and the FI parameters uniquely specify the four-dimensional $\mathcal{N}=2$ gauged supergravity Lagrangian and BPS variations.

Now, we can proceed with the explicit reduction of the line element (4.10) on the Hopf fibres of $S^{3}$ viewed as a U(1) bundle over $S^{2} \cong \mathbb{C P}^{1}$. We thus identify $x^{5}$ with $\chi$. The four-dimensional solution takes the form ${ }^{5}$

$$
\begin{array}{rlrl}
d s_{(4)}^{2} & =-e^{2 U} d \tilde{\tau}^{2}+e^{-2 U} d r^{2}+e^{2(V-U)}\left(d \vartheta^{2}+\sin ^{2} \vartheta d \varphi^{2}\right), \\
A_{(4)}^{0} & =\tilde{q}_{(4)}^{0}(r) d \tilde{\tau}-\cos \vartheta d \varphi, & A_{(4)}^{I}=\tilde{q}_{(4)}^{I}(r) d \tilde{\tau},
\end{array}
$$

where

$$
\begin{aligned}
e^{U} & =\frac{\sqrt{2}}{R_{\mathrm{AdS}_{2}} R_{S^{2}}^{1 / 2} v^{1 / 4}} r, & e^{V} & =\frac{R_{S^{2}}}{2 R_{\mathrm{AdS}_{2}}} r \\
\tilde{q}_{(4)}^{0}(r) & =-\frac{2 \alpha}{R_{\mathrm{AdS}_{2}}^{2} R_{S^{2}} v^{1 / 2}} r, & \tilde{q}_{(4)}^{I}(r) & =-\frac{2\left(e^{I}-f^{I} \alpha\right)}{R_{\mathrm{AdS}_{2}}^{2} R_{S^{2}} v^{1 / 2}} r .
\end{aligned}
$$

The complex scalars are given by

$$
z^{I}=-f^{I}+\frac{i}{2} R_{S^{2}} v^{1 / 2} L^{I}=-f^{I}+\frac{i}{2} \frac{v^{1 / 2} \gamma_{3}^{1 / 2}}{\mu_{I}} .
$$

Employing eq. (B.20) we can compute the conserved electric charges. After some work they read

$$
\begin{aligned}
& q_{0}=\frac{g}{8}\left(\gamma_{2}+2 g^{2} \gamma_{3}\right)=\frac{G_{\mathrm{N}}^{(5)}}{\pi} J_{\phi}, \\
& q_{I}=-\frac{1}{4}\left[\mu_{I}+\frac{g^{2}}{2}\left(\gamma_{2}-\frac{2 \gamma_{3}}{\mu_{I}}\right)\right]=-\frac{G_{\mathrm{N}}^{(5)}}{\pi} Q_{I} .
\end{aligned}
$$

This is in agreement with [52]. The magnetic charges of the four-dimensional solution can be directly read off the spherical components of the gauge fields $A_{(4)}^{\Lambda}(4.15)$,

$$
p^{0}=1, \quad p^{I}=0 .
$$

The entropy of the four-dimensional black hole precisely equals the entropy of the rotating black hole in five dimensions,

$$
S_{\mathrm{BH}}^{(4)}=\frac{\operatorname{Area}^{(4)}}{4 G_{\mathrm{N}}^{(4)}}=\frac{\pi e^{2(V-U)}}{G_{\mathrm{N}}^{(4)}}=\frac{\pi^{2} R_{S^{2}}^{3} v^{1 / 2}}{2 G_{\mathrm{N}}^{(5)}}=S_{\mathrm{BH}}^{(5)},
$$

upon using the standard relation

$$
\frac{1}{G_{\mathrm{N}}^{(4)}}=\frac{4 \pi}{G_{\mathrm{N}}^{(5)}} .
$$

\footnotetext{
${ }^{5}$ We have rescaled the time coordinate, $\tilde{\tau} \equiv-R_{S^{2}} R_{\mathrm{AdS}_{2}} \sqrt{v} \tau / 2$, in order to put the AdS 2 part of the metric in the canonical coordinates.
} 


\subsection{Attractor mechanism in four dimensions}

Let us define the central charge of the black hole $\mathcal{Z}$ and the superpotential $\mathcal{L}$,

$$
\mathcal{Z}=e^{\mathcal{K} / 2}\left(q_{\Lambda} X^{\Lambda}-p^{\Lambda} \mathcal{F}_{\Lambda}\right), \quad \mathcal{L}=e^{\mathcal{K} / 2}\left(g_{\Lambda} X^{\Lambda}-g^{\Lambda} \mathcal{F}_{\Lambda}\right)
$$

The BPS equations for the near-horizon solution (B.13) with constant scalar fields $z^{i}$ imply that $[29]:{ }^{6}$

$$
\mathcal{Z}+2 i e^{2(V-U)} \mathcal{L}=0, \quad D_{j}\left(\mathcal{Z}+2 i e^{2(V-U)} \mathcal{L}\right)=0,
$$

which can be rewritten as

$$
\partial_{j} \frac{\mathcal{Z}}{\mathcal{L}}=0, \quad i \frac{\mathcal{Z}}{\mathcal{L}}=2 e^{2(V-U)} .
$$

Here, $D_{j}=\partial_{j}+\frac{1}{2} \partial_{j} \mathcal{K}$ with $\mathcal{K}$ being the Kähler potential [see eq. (B.4)]. Therefore, the complex scalar fields $z^{i}$ are fixed at the horizon such that the quantity $i \frac{\mathcal{Z}}{\mathcal{L}}$ has a critical point on $\mathcal{M}$ and then its value is proportional to the Bekenstein-Hawking entropy of the BPS black hole.

We can extremize the quantity $i \frac{\mathcal{Z}}{\mathcal{L}}$ under the following gauge fixing constraint, which precisely corresponds to (1.3),

$$
g_{0} X^{0}+\sum_{I=1}^{3} X^{I}=1
$$

where we plugged in the explicit values for the FI parameters, i.e., $g_{1}=g_{2}=g_{3}=1$. The real sections $X^{\Lambda}$ are constrained in the range $0<X^{\Lambda}<1$. We find that

$$
\partial_{I}\left[\sum_{I=1}^{3} X^{I}\left(q_{I}-\frac{q_{0}}{g_{0}}\right)+\frac{q_{0}}{g_{0}}-\frac{g_{0}^{2} X^{1} X^{2} X^{3}}{\left(1-X^{1}-X^{2}-X^{3}\right)^{2}}\right]=0, \quad \text { for } \quad I=1,2,3,
$$

where $\partial_{I} \equiv \partial / \partial X^{I}$. The sections at the horizon are obtained from

$$
X^{0}=\frac{1}{g_{0}\left(1+z^{1}+z^{2}+z^{3}\right)}, \quad X^{1,2,3}=\frac{z^{1,2,3}}{1+z^{1}+z^{2}+z^{3}} .
$$

We are now in a position to determine the value of the FI parameter $g_{0}$. Partial topological A-twist along $S^{2}$, embedding the spacetime holonomy group into the R-symmetry, ensures that $\mathcal{N}=2$ supersymmetry is preserved in four dimensions [53]. The twisting amounts to an identification of the spin connection with the R-symmetry so that one of the SUSY parameters becomes a scalar. This leads to the following Dirac-like quantization condition [28-30]:

$$
g_{\Lambda} p^{\Lambda}=1=g_{0} p^{0},
$$

which fixes the value of $g_{0}=1$. It is straightforward to check that, substituting the values for the physical scalars at the horizon (4.17), the charges (4.18), and setting $g_{0}=1$,

\footnotetext{
${ }^{6}$ From comparing (4.23) with equations (3.5) and (3.8) in [29], we see that they differ by a factor of 2. This is due to our different convention for the action (see footnote 10).
} 
eq. (4.26) is fulfilled. The scalars $\bar{z}^{i}\left(r_{\mathrm{h}}\right)$ at the horizon are determined in terms of the black hole charges $q_{I}$ by virtue of the attractor equations:

$$
q_{I}-q_{0}=\left(2+\frac{1}{\bar{z}^{I}}\right) \bar{z}^{1} \bar{z}^{2} \bar{z}^{3} .
$$

The value of $i \frac{\mathcal{Z}}{\mathcal{L}}$ at the critical point yields,

$$
\left.i \frac{\mathcal{Z}}{\mathcal{L}}\right|_{\text {crit }}\left(q_{\Lambda}\right)=2 e^{2(V-U)}=\frac{2 G_{\mathrm{N}}^{(4)}}{\pi} S_{\mathrm{BH}}^{(4)}\left(q_{\Lambda}\right) .
$$

The holding of the four-dimensional BPS attractor mechanism for the dimensionally reduced near-horizon geometry (4.15) proves that the dimensional reduction preserves the full amount of supersymmetries originally present in five dimensions.

Due to the very suggestive form of the attractor equations (4.26) it is now not hard to compare them with the five-dimensional extremization.

\subsection{Comparison with five-dimensional extremization}

Consider the quantity $E$ in (3.3) rewritten in terms of the chemical potentials for $J^{ \pm}$and $Q_{1,2,3}$. Recall that we are focusing on the case with equal angular momenta, i.e., $J_{\psi}=J_{\phi}$ (so $J^{-}=0$ ). Extremizing (3.5) with respect to $X_{-}^{0}$ fixes the value of $X_{-}^{0}=0$. Thus, the black hole entropy is obtained by extremizing the quantity

$$
\left.\mathcal{S}\right|_{J^{-}=0}=\frac{2 \pi^{2} i}{G_{\mathrm{N}}^{(5)}} \frac{X^{1} X^{2} X^{3}}{\left(X_{+}^{0}\right)^{2}}+2 \pi i \sum_{I=1}^{3} Q_{I} X^{I}-\pi i J^{+} X_{+}^{0},
$$

subject to the constraint (3.6). Identifying $X^{0}$ in (4.26) with $X_{+}^{0}$ in (4.31), and using $g_{0}=1$ together with (4.18), we find that the extremization of $\mathcal{S}$ corresponds to the fourdimensional attractor mechanism on the gravity side and they lead to the same entropy.

\section{Discussion and future directions}

We have shown that the entropy of a supersymmetric rotating black hole in $\mathrm{AdS}_{5}$ with electric charges $Q_{I}(I=1,2,3)$ and angular momenta $J_{\phi} \equiv J_{1}, J_{\psi} \equiv J_{2}$ can be obtained as the Legendre transform of the quantity $(-E)$ in (1.2),

$$
\mathcal{S}\left(Q_{I}, J_{i}\right)=-E\left(\Delta_{I}, \omega_{i}\right)+\left.2 \pi i\left(\sum_{I=1}^{3} Q_{I} \Delta_{I}-\sum_{i=1}^{2} J_{i} \omega_{i}\right)\right|_{\bar{\Delta}_{I}, \bar{\omega}_{i}},
$$

where $\bar{\Delta}_{I}$ and $\bar{\omega}_{i}$ are the extrema of the functional on the right hand side.

The result is quite intriguing and deserves a better explanation and understanding. We leave a more careful analysis for the future. For the moment, let us just make few preliminary observations.

The quantity $E$ can be interpreted as a combination of 't Hooft anomaly polynomials that arise studying the partition function $Z_{\mathcal{N}=4}\left(\Delta_{I}, \omega_{i}\right)$ on $S^{3} \times S^{1}$ or the superconformal 
index $I\left(\Delta_{I}, \omega_{i}\right)$ for $\mathcal{N}=4 \mathrm{SYM}[13,16]$. Some explicit expressions are given in appendix C. In particular, $E$ is formally equal to the supersymmetric Casimir energy of $\mathcal{N}=4 \mathrm{SYM}$ as a function of the chemical potentials (see for example equation (4.50) in [13] and appendix C). However, this analogy is only formal since we are imposing the constraint (1.3). Chemical potentials are only defined modulo 1 , so the constraint to be imposed on them also suffers from angular ambiguities. Consistency of the index and partition function just requires $\sum_{I=1}^{3} \Delta_{I}+\sum_{i=1}^{2} \omega_{i} \in \mathbb{Z}$. To recover the known expressions for the supersymmetric Casimir energy and for consistency with gauge anomaly cancellations [12, 13], one needs to impose $\sum_{I=1}^{3} \Delta_{I}+\sum_{i=1}^{2} \omega_{i}=0$, and this contrasts with (1.3).

It would be tempting to interpret the Legendre transform (5.1) as a result of the saddle-point approximation of a Laplace integral of $Z_{\mathcal{N}=4}$ in the limit of large charges (large $N) .{ }^{7}$ Ignoring angular ambiguities, $E$ is the leading contribution at order $N^{2}$ of the logarithm of the partition function $Z_{\mathcal{N}=4}$ on $S^{3} \times S^{1}$. Indeed, $\log Z_{\mathcal{N}=4}=-E+$ $\log I[10-16]$ and the index is a quantity independent of $N$ for generic values for the chemical potentials [6]. In these terms, the result would be completely analogous to the connection between asymptotically $\mathrm{AdS}_{4} \times S^{7}$ black hole entropy and the topologically twisted index of ABJM [17, 18].

The appearance of the supersymmetric Casimir energy can be surprising since the entropy counts the degeneracy of ground states of the system. However, the dimensional reduction to four dimensions performed in section 4 offers a different perspective on this point. The dimensionally reduced black hole is static but not asymptotically AdS. Let us assume that we can still use holography. In the dimensional reduction, a magnetic flux $p^{0}$ is turned on for the graviphoton. This means that supersymmetry is preserved with a topological twist. The same should be true for the boundary theory. It is then tempting to speculate that, upon dimensional reduction, the partition function $Z_{\mathcal{N}=4}$ becomes the topologically twisted index of the boundary three-dimensional theory [19]. The supersymmetric Casimir energy, which is the leading contribution of $\log Z_{\mathcal{N}=4}$ at large $N$ then becomes the leading contribution of the three-dimensional topologically twisted index and the latter is known to correctly account for the microstates of four-dimensional black holes [17, 18].

The above discussion ignores completely the angular ambiguities and the role of the constraint (1.3), which should be further investigated. For sure, the result of the extremization of $E$ is complex and lies in the region where the chemical potentials satisfy (1.3). Unfortunately, we are not aware of a general discussion of the possible regularizations of $Z_{\mathcal{N}=4}$ that takes into account the angular ambiguities. Moreover, there is some recent claim $[54,55]$ of the presence of an anomaly in the supersymmetry transformations leading to a modification of the BPS condition in gravity that would be interesting to investigate further in this context.

Both the constraint (1.3) and the analogous of the more traditional one $\sum_{I=1}^{3} \Delta_{I}+$ $\sum_{i=1}^{2} \omega_{i}=0$ have been used in the literature to explore different features of $Z_{\mathcal{N}=4}$ or the index. The traditional constraint has been used in the analysis of the high temperature limit of the index $[56,57]$ (see also $[58,59]$ ) and in the study of factorization properties [60]. On

\footnotetext{
${ }^{7}$ We are ignoring here potential sign ambiguities in the definition of charges.
} 
the other hand, the importance of (1.3) has been stressed in [16] where the constraint has been used to extract the supersymmetric Casimir energy directly from the superconformal index. ${ }^{8}$ See appendix $\mathrm{C}$ for more details. In the low temperature limit, which can be obtained by rescaling $\Delta_{I} \rightarrow \beta \Delta_{I}, \omega_{i} \rightarrow \beta \omega_{i}$ and taking large $\beta$, the angular ambiguity in the constraint disappears.

Finally, it is worth mentioning that angular ambiguities also played a prominent role in the evaluation of the saddle-point for the topologically twisted index of the ABJM theory and the comparison with the entropy of $\mathrm{AdS}_{4}$ black holes [17].

All this is quite speculative and we hope to come back with more precise statements in the future. There are also other directions to investigate. Let us mention some of them.

Attractor mechanism in five dimensions: it is known that in five-dimensional $\mathcal{N}=2$ ungauged supergravity the near-horizon geometry of an extremal BPS black hole is governed by the attractor mechanism [27,62-64]. That is, the values of the scalar fields at the horizon are fixed by black hole charges, and the area of the black hole horizon is given in terms of the extremal value of the central charge $\overline{\mathcal{Z}}$ in moduli space and the angular momentum $J$,

$$
\text { Area }^{(5)}=\frac{\pi^{2}}{3} \sqrt{\overline{\mathcal{Z}}^{3}-J^{2}} .
$$

It would be interesting to find analogous results in five-dimensional gauged supergravity ${ }^{9}$ and see if we can recover the extremization principle (5.1).

Rotating attractors in four dimensions: as explained in section 4 we only understand well the static attractor equations in four dimensions. It is natural to extend this analysis to rotating cases, which will correspond to the refinement by angular momentum in the dual field theory. We would have already had an example of a rotating attractor if we were to consider the dimensional reduction of the generic BPS black hole with $J_{\psi} \neq J_{\phi}$.

Dimensional reduction of black strings in five dimensions: similarly to the reduction of BPS black holes from five to four dimensions, one could perform a reduction between five-dimensional BPS black strings and four-dimensional BPS black holes, as it was already done in [40]. Given the recent results [68] on the topologically twisted index for $\mathcal{N}=1$ supersymmetric theories on $S^{2} \times T^{2}$, this could imply a relation between the $c$-extremization of the two-dimensional SCFTs $[69,70]$ and the $\mathcal{I}$-extremization principle of $[17,18]$.

\section{Acknowledgments}

We would like to thank Arash Arabi Ardehali, Francesco Benini, Nikolay Bobev, Sara Pasquetti, Vyacheslav S. Spiridonov and Chiara Toldo for useful discussions and comments. AZ is partially supported by the INFN and ERC-STG grant 637844-HBQFTNCER. SMH is supported in part by INFN. KH is supported in part by the Bulgarian NSF grant DN08/3.

\footnotetext{
${ }^{8}$ Interestingly, the same constraint is also used in relating the universal part of supersymmetric Rényi entropy to an equivariant integral of the anomaly polynomial [61].

${ }^{9}$ First-order flow equations for stationary black brane solutions and magnetically charged black strings in five-dimensional $\mathcal{N}=2$ gauged supergravity were analyzed in [65-67].
} 


\section{A Five-dimensional $\mathcal{N}=2$ gauged supergravity}

The theory we shall be considering, following the conventions of [48], is the five-dimensional $\mathcal{N}=2$ Fayet-Iliopoulos (FI) gauged supergravity coupled to $n_{\mathrm{V}}$ vector multiplets. It is based on a homogeneous real cubic polynomial

$$
\mathcal{V}\left(L^{I}\right)=\frac{1}{6} C_{I J K} L^{I} L^{J} L^{K},
$$

where $I, J, K=1, \ldots, n_{\mathrm{V}}$ and $C_{I J K}$ is a fully symmetric third-rank tensor appearing in the Chern-Simons term. Here, $L^{I}\left(\varphi^{i}\right)$ are real scalars satisfying the constraint $\mathcal{V}=1$. The action for the bosonic sector reads

$$
\begin{aligned}
S^{(5)}= & \int_{\mathbb{R}^{4,1}}\left[\frac{1}{2} R^{(5)} \star_{5} 1-\frac{1}{2} G_{I J} \mathrm{~d} L^{I} \wedge \star_{5} \mathrm{~d} L^{J}-\frac{1}{2} G_{I J} F^{I} \wedge \star_{5} F^{J}\right. \\
& \left.-\frac{1}{12} C_{I J K} F^{I} \wedge F^{J} \wedge A^{K}+\chi^{2} V \star_{5} 1\right],
\end{aligned}
$$

where $R^{(5)}$ is the Ricci scalar, $F^{I} \equiv \mathrm{d} A^{I}$ is the Maxwell field strength, and $G_{I J}$ can be written in terms of $\mathcal{V}$,

$$
G_{I J}=-\left.\frac{1}{2} \partial_{I} \partial_{J} \log \mathcal{V}\right|_{\mathcal{V}=1}
$$

We also set $8 \pi G_{\mathrm{N}}^{(5)}=1$. Furthermore, it is convenient to define

$$
L_{I} \equiv \frac{1}{6} C_{I J K} L^{J} L^{K}
$$

Therefore, we find that

$$
G_{I J}=\frac{9}{2} L_{I} L_{J}-\frac{1}{2} C_{I J K} L^{K}, \quad L^{I} L_{I}=1,
$$

and

$$
L_{I}=\frac{2}{3} G_{I J} L^{J}, \quad L^{I}=\frac{3}{2} G^{I J} L_{J},
$$

where $G_{I K} G^{K J}=\delta_{J}^{I}$. The inverse of $G_{I J}$ is given by

$$
G^{I J}=2 L^{I} L^{J}-6 C^{I J K} L_{K},
$$

where $C^{I J K} \equiv C_{I J K}$. We then have

$$
L^{I}=\frac{9}{2} C^{I J K} L_{J} L_{K} .
$$

The metric on the scalar manifold is defined by

$$
g_{i j}=\left.\partial_{i} L^{I} \partial_{j} L^{J} G_{I J}\right|_{\mathcal{V}=1},
$$

and the scalar potential reads

$$
V(L)=V_{I} V_{J}\left(6 L^{I} L^{J}-\frac{9}{2} g^{i j} \partial_{i} L^{I} \partial_{j} L^{J}\right) .
$$


Here, $V_{I}$ are FI constants which are related to the vacuum value $\bar{L}_{I}$ of the scalars $L_{I}$,

$$
\bar{L}_{I}=\xi^{-1} V_{I}
$$

where $\xi^{3}=\frac{9}{2} C^{I J K} V_{I} V_{J} V_{K}$ and the $\mathrm{AdS}_{5}$ radius of curvature is given by $g^{-1} \equiv(\xi \chi)^{-1}$. A useful relation of very special geometry is,

$$
g^{i j} \partial_{i} L^{I} \partial_{j} L^{J}=G^{I J}-\frac{2}{3} L^{I} L^{J}
$$

Thus,

$$
V(L)=9 V_{I} V_{J}\left(L^{I} L^{J}-\frac{1}{2} G^{I J}\right)
$$

\section{B Four-dimensional $\mathcal{N}=2$ gauged supergravity}

We consider the four-dimensional $\mathcal{N}=2$ FI-gauged supergravity with a holomorphic prepotential

$$
\mathcal{F}\left(X^{\Lambda}\right)=-\frac{1}{6} \frac{C_{I J K} X^{I} X^{J} X^{K}}{X^{0}}
$$

where $X^{\Lambda}$ represent the symplectic sections and $\Lambda=0,1,2,3$. The physical scalars $z^{I}$ $(I=1,2,3)$ belonging to the vector multiplets are given by

$$
z^{I}=\frac{X^{I}}{X^{0}}
$$

and parameterize a special Kähler manifold $\mathcal{M}$ of complex dimension $n_{\mathrm{V}}$ with metric

$$
g_{i \bar{j}}=\partial_{i} \partial_{\bar{j}} \mathcal{K}(z, \bar{z})
$$

Here, $\mathcal{K}(z, \bar{z})$ is the Kähler potential and it reads

$$
e^{-\mathcal{K}(z, \bar{z})}=i\left(\bar{X}^{\Lambda} \mathcal{F}_{\Lambda}-X^{\Lambda} \overline{\mathcal{F}}_{\Lambda}\right)
$$

where $\mathcal{F}_{\Lambda} \equiv \partial_{\Lambda} \mathcal{F}$. Plugging (B.1) into (B.4) we find that

$$
e^{-\mathcal{K}(z, \bar{z})}=\frac{4 i}{3} C_{I J K} \operatorname{Im} z^{I} \operatorname{Im} z^{J} \operatorname{Im} z^{K}=8 e^{-6 \phi},
$$

where due to the symmetries of the theory we can set $X^{0}=1$. In the last equality we used eq. (4.13). Substituting (B.5) into (B.3) and using

$$
\frac{\partial}{\partial z^{I}}=\frac{1}{2}\left(\frac{\partial}{\partial \mathbb{R e} z^{I}}-i \frac{\partial}{\partial \operatorname{Im} z^{I}}\right), \quad \frac{\partial}{\partial \bar{z}^{I}}=\frac{1}{2}\left(\frac{\partial}{\partial \operatorname{Re} z^{I}}+i \frac{\partial}{\partial \operatorname{Im} z^{I}}\right),
$$

we can write the Kähler metric as

$$
\begin{aligned}
g_{I J} & =-\frac{1}{4} \frac{\partial}{\partial \operatorname{Im} z^{I}} \frac{\partial}{\partial \operatorname{Im} z^{J}} \log \left(\frac{4 i}{3} C_{I J K} \operatorname{Im} z^{I} \operatorname{Im} z^{J} \operatorname{Im} z^{K}\right) \\
& =-\frac{1}{4 e^{-6 \phi}}\left(C_{I J}-\frac{C_{I} C_{J}}{4 e^{-6 \phi}}\right) .
\end{aligned}
$$


Here, we introduced the following notation

$$
C_{I J}=C_{I J K} \operatorname{Im} z^{K}, \quad C_{I}=C_{I J K} \operatorname{Im} z^{J} \mathbb{I m} z^{K} .
$$

The action of the bosonic part of the supergravity reads $[71,72]^{10}$

$$
\begin{aligned}
S^{(4)}= & \int_{\mathbb{R}^{3,1}}\left[\frac{1}{2} R^{(4)} \star_{4} 1+\frac{1}{4} \operatorname{Im} \mathcal{N}_{\Lambda \Sigma} F^{\Lambda} \wedge \star_{4} F^{\Sigma}+\frac{1}{4} \mathbb{R e} \mathcal{N}_{\Lambda \Sigma} F^{\Lambda} \wedge F^{\Sigma}\right. \\
& \left.-g_{i \bar{j}} D z^{i} \wedge \star_{4} D \bar{z}^{\bar{j}}-V(z, \bar{z}) \star_{4} 1\right],
\end{aligned}
$$

where $\Lambda, \Sigma=0,1, \ldots, n_{\mathrm{V}}$ and $i, \bar{j}=1, \ldots, n_{\mathrm{V}}$. Note that we already set $8 \pi G_{\mathrm{N}}^{(4)}=1$. Here, $V$ is the scalar potential of the theory,

$$
V(z, \bar{z})=2 g^{2}\left(U^{\Lambda \Sigma}-3 e^{\mathcal{K}} \bar{X}^{\Lambda} X^{\Sigma}\right) \xi_{\Lambda} \xi_{\Sigma}
$$

where $\xi_{\Lambda}$ are the constant quaternionic moment maps known as FI parameters and

$$
U^{\Lambda \Sigma}=-\frac{1}{2}(\operatorname{Im} \mathcal{N})^{-1 \mid \Lambda \Sigma}-e^{\mathcal{K}} \bar{X}^{\Lambda} X^{\Sigma}
$$

The matrix $\mathcal{N}_{\Lambda \Sigma}$ of the gauge kinetic term is a function of the vector multiplet scalars and is given by

$$
\mathcal{N}_{\Lambda \Sigma}=\overline{\mathcal{F}}_{\Lambda \Sigma}+2 i \frac{\operatorname{Im} \mathcal{F}_{\Lambda \Delta} \operatorname{Im} \mathcal{F}_{\Sigma \Theta} X^{\Delta} X^{\Theta}}{\operatorname{Im} \mathcal{F}_{\Delta \Theta} X^{\Delta} X^{\Theta}}
$$

In this paper we focus on black holes with the near-horizon geometry $\mathrm{AdS}_{2} \times S^{2}$. The ansatz for the metric and gauge fields is

$$
\begin{aligned}
d s^{2} & =-e^{2 U} d \tau^{2}+e^{-2 U} d r^{2}+e^{2(V-U)}\left(d \vartheta^{2}+\sin ^{2} \vartheta d \varphi^{2}\right) \\
A^{\Lambda} & =\tilde{q}^{\Lambda}(r) d \tau-p^{\Lambda}(r) \cos \vartheta d \varphi
\end{aligned}
$$

The black hole magnetic and electric charges are then given by [29, 73]

$$
\begin{aligned}
p^{\Lambda} & \equiv \frac{1}{4 \pi} \int_{S^{2}} F^{\Lambda}, \\
q_{\Lambda} & \equiv \frac{1}{4 \pi} \int_{S^{2}} G_{\Lambda}=e^{2(V-U)} \operatorname{Im} \mathcal{N}_{\Lambda \Sigma} \tilde{q}^{\Sigma \Sigma}+\mathbb{R e} \mathcal{N}_{\Lambda \Sigma} p^{\Sigma},
\end{aligned}
$$

where we defined the symplectic-dual gauge field strength,

$$
G_{\Lambda} \equiv 2 \frac{\delta \mathcal{L}}{\delta F^{\Lambda}}=\mathbb{I m} \mathcal{N}_{\Lambda \Sigma \star_{4}} F^{\Sigma}+\operatorname{Re} \mathcal{N}_{\Lambda \Sigma} F^{\Sigma}
$$

\footnotetext{
${ }^{10}$ We follow the conventions of [48], which is different from [71] by factors of two in the gauge kinetic terms and the scalar potential $V(z, \bar{z})$. One can swap between the conventions by rescaling the fourdimensional metric $g_{\mu \nu} \rightarrow \frac{1}{2} g_{\mu \nu}$ and then multiplying the action by 2 . This will modify the definition of the symplectic-dual gauge field strength $G_{\Lambda}$ by a factor of 2 , see eq. (B.15).
} 
such that $\left(F^{\Lambda}, G_{\Lambda}\right)$ transforms as a $\left(2, n_{\mathrm{V}}+2\right)$ symplectic vector under electric-magnetic duality transformations. Employing eq. (B.12) we find that

$$
\begin{array}{ll}
\operatorname{Im} \mathcal{N}_{I J}=\mathcal{G}_{I J}, & \mathbb{R e} \mathcal{N}_{I J}=-C_{I J K} \operatorname{Re} z^{K}, \\
\operatorname{Im} \mathcal{N}_{I 0}=-\mathcal{G}_{I J} \operatorname{Re} z^{J}, & \operatorname{Re} \mathcal{N}_{I 0}=+\frac{1}{2} C_{I J K} \operatorname{Re} z^{J} \operatorname{Re} z^{K}, \\
\operatorname{Im} \mathcal{N}_{00}=-\left(e^{-6 \phi}-\mathcal{G}_{I J} \mathbb{R e} z^{I} \operatorname{Re} z^{J}\right), & \mathbb{R e} \mathcal{N}_{00}=-\frac{1}{3} C_{I J K} \operatorname{Re} z^{I} \operatorname{Re} z^{J} \operatorname{Re} z^{K},
\end{array}
$$

where we defined

$$
\mathcal{G}_{I J} \equiv C_{I J}-\frac{C_{I} C_{J}}{4 e^{-6 \phi}}
$$

Therefore, the electric charges read

$$
\begin{aligned}
q_{0}= & -e^{2(V-U)} \tilde{q}^{0}\left[e^{-6 \phi}+\mathcal{G}_{I J} \operatorname{Re} z^{J}\left(\frac{\tilde{q}^{\prime I}}{\tilde{q}^{0}}-\mathbb{R e} z^{I}\right)\right] \\
& +\frac{p^{0}}{2} C_{I J K} \mathbb{R e} z^{J} \mathbb{R e} z^{K}\left(\frac{p^{I}}{p^{0}}-\frac{2}{3} \mathbb{R e} z^{I}\right), \\
q_{I}= & e^{2(V-U)} \tilde{q}^{0} \mathcal{G}_{I J}\left(\frac{\tilde{q}^{J}}{\tilde{q}^{\prime 0}}-\mathbb{R e} z^{J}\right)-p^{0} C_{I J K} \mathbb{R e} z^{K}\left(\frac{p^{J}}{p^{0}}-\frac{1}{2} \mathbb{R e} z^{J}\right) .
\end{aligned}
$$

In the gauged STU model $\left(n_{\mathrm{V}}=3\right)$ the only nonvanishing intersection numbers are $C_{123}=1$ (and cyclic permutations). Hence,

$$
\mathcal{G}_{I J}=\left\{\begin{array}{ll}
-\frac{\mathbb{m} z^{1} \mathbb{m} z^{2} \mathbb{I m} z^{3}}{\left(\mathbb{I m} z^{I}\right)^{2}} & \text { if } I=J \\
0 & \text { otherwise }
\end{array},\right.
$$

and

$$
\begin{aligned}
q_{0}= & -e^{2(V-U)} \tilde{q}^{00} \operatorname{Im} \Pi\left[1-\sum_{I=1}^{3} \frac{\mathbb{R e} z^{I}}{\left(\operatorname{Im} z^{I}\right)^{2}}\left(\frac{\tilde{q}^{I}}{\tilde{q}^{\prime 0}}-\mathbb{R e} z^{I}\right)\right] \\
& -2 p^{0} \operatorname{Re} \Pi+\sum_{\substack{I<J \\
(\neq K)}} \operatorname{Re} z^{I} \mathbb{R e} z^{J} p^{K}, \\
q_{I}= & -e^{2(V-U)} \tilde{q}^{\prime 0} \frac{\mathbb{I m} \Pi}{\left(\mathbb{I m} z^{I}\right)^{2}}\left(\frac{\tilde{q}^{I}}{\tilde{q}^{\prime 0}}-\mathbb{R e} z^{I}\right)+\frac{\mathbb{R e} \Pi}{\operatorname{Re} z^{I}}\left(p^{0}-\sum_{J(\neq I)} \frac{p^{J}}{\operatorname{Re} z^{J}}\right),
\end{aligned}
$$

where we employed the following notation

$$
\operatorname{Im} \Pi \equiv \operatorname{Im} z^{1} \operatorname{Im} z^{2} \operatorname{Im} z^{3}, \quad \quad \mathbb{R e} \Pi \equiv \mathbb{R e} z^{1} \mathbb{R e} z^{2} \operatorname{Re} z^{3} .
$$

\section{Generalities about the supersymmetric Casimir energy}

The partition function of an $\mathcal{N}=1$ supersymmetric gauge theory with a non-anomalous $\mathrm{U}(1)_{R}$ symmetry on a Hopf surface $\mathcal{H}_{p, q} \simeq S^{3} \times S^{1}$, with a complex structure characterized by two parameters $p, q$, can be written as

$$
Z\left[\mathcal{H}_{p, q}\right]=e^{-E_{\mathrm{SUSY}}} I(p, q) .
$$


Here, $I(p, q)$ is the superconformal index $[6,9]$

$$
I(p, q)=\operatorname{Tr}(-1)^{F} p^{h_{1}+r / 2} q^{h_{2}+r / 2},
$$

where $h_{1}$ and $h_{2}$ are the generators of rotation in orthogonal planes and $r$ is the superconformal R-symmetry. $E_{\mathrm{SUSY}}$ is the supersymmetric Casimir energy [10-12],

$$
E_{\mathrm{SUSY}}\left(b_{1}, b_{2}\right)=\frac{4 \pi}{27} \frac{\left(\left|b_{1}\right|+\left|b_{2}\right|\right)^{3}}{\left|b_{1}\right|\left|b_{2}\right|}(3 c-2 a)-\frac{4 \pi}{3}\left(\left|b_{1}\right|+\left|b_{2}\right|\right)(c-a),
$$

where $p=e^{-2 \pi\left|b_{1}\right|}, q=e^{-2 \pi\left|b_{2}\right|}$, and $a, c$ are the central charges of the four-dimensional $\mathcal{N}=1$ theory. We can extrapolate this result to include flavor symmetries by considering $a$ and $c$ as trial central charges, depending on a set of chemical potentials $\hat{\Delta}_{I}$,

$$
a\left(\hat{\Delta}_{I}\right)=\frac{9}{32} \operatorname{Tr} R\left(\hat{\Delta}_{I}\right)^{3}-\frac{3}{32} \operatorname{Tr} R\left(\hat{\Delta}_{I}\right), \quad c\left(\hat{\Delta}_{I}\right)=\frac{9}{32} \operatorname{Tr} R\left(\hat{\Delta}_{I}\right)^{3}-\frac{5}{32} \operatorname{Tr} R\left(\hat{\Delta}_{I}\right),
$$

where $R$ is a choice of $\mathrm{U}(1)_{R}$ symmetry and the trace is over all fermions in the theory. The supersymmetric Casimir energy can be also interpreted as the vacuum expectation value $\left\langle H_{\text {susy }}\right\rangle$ of the Hamiltonian which generates time translations [12]. It can also be obtained by integrating anomaly polynomials in six dimensions [13]. In particular, the supersymmetric Casimir energy for $\mathcal{N}=4$ super Yang-Mills (SYM) theory with $\mathrm{SU}(N)$ gauge group, where $a=c$, reads ${ }^{11}$

$$
E_{\mathrm{SUSY}}=\frac{\pi}{8}\left(N^{2}-1\right) \frac{\left(\left|b_{1}\right|+\left|b_{2}\right|\right)^{3}}{\left|b_{1}\right|\left|b_{2}\right|} \hat{\Delta}_{1} \hat{\Delta}_{2} \hat{\Delta}_{3}
$$

where $\hat{\Delta}_{1,2,3}$ are the chemical potentials for the Cartan generators of the R-symmetry, fulfilling the constraint

$$
\hat{\Delta}_{1}+\hat{\Delta}_{2}+\hat{\Delta}_{3}=2 \text {. }
$$

We can rewrite eq. (C.5) in the notation used in the main text as

$$
E_{\mathrm{SUSY}}=-i \pi\left(N^{2}-1\right) \frac{\Delta_{1} \Delta_{2} \Delta_{3}}{\omega_{1} \omega_{2}},
$$

where we defined $\Delta_{I}=i\left(\left|b_{1}\right|+\left|b_{2}\right|\right) \hat{\Delta}_{I} / 2$ and $\omega_{i}=-i\left|b_{i}\right|$. They satisfy the constraint

$$
\Delta_{1}+\Delta_{2}+\Delta_{3}+\omega_{1}+\omega_{2}=0 .
$$

When extended to the complex plane $\Delta_{I}$ and $\omega_{i}$ are defined modulo 1.

The constraints (C.8) and (1.3) are closely related to the absence of pure and mixed gauge anomalies. Let us briefly see why. Consider again a generic $\mathcal{N}=1$ supersymmetric gauge theory. The constraint (C.8) is modified to

$$
\sum_{I \in W_{a}} \Delta_{I}+\sum_{i=1}^{2} \omega_{i}=0,
$$

\footnotetext{
${ }^{11}$ The R-symmetry 't Hooft anomalies for $\mathcal{N}=4 \mathrm{SYM}$ are given by $\operatorname{Tr} R\left(\hat{\Delta}_{I}\right)=\left(N^{2}-1\right)\left[\sum_{I=1}^{3}\left(\hat{\Delta}_{I}-1\right)+\right.$ $1]=0$ and $\operatorname{Tr} R\left(\hat{\Delta}_{I}\right)^{3}=\left(N^{2}-1\right)\left[\sum_{I=1}^{3}\left(\hat{\Delta}_{I}-1\right)^{3}+1\right]=3\left(N^{2}-1\right) \hat{\Delta}_{1} \hat{\Delta}_{2} \hat{\Delta}_{3}$. The $\hat{\Delta}_{I}$ are the R-symmetries of the three adjoint chiral multiplets of $\mathcal{N}=4$ SYM and satisfy (C.6).
} 
where $\Delta_{I}$ is the chemical potential for the $I$-th field and the sum is restricted to the fields entering the superpotential term $W_{a}$. There is one constraint for each monomial $W_{a}$ in the superpotential of the theory. The path integral on $S^{3} \times S^{1}$ localizes to a matrix model where one-loop determinants must be regularized. $E_{\text {SUSY }}$ arises from the following regularization factors $[12,13]$

$$
\Psi(u)=i \pi \frac{\left(\sum_{i=1}^{2} \omega_{i}\right)^{3}}{24 \omega_{1} \omega_{2}}\left[(\hat{u}-1)^{3}-\frac{\sum_{i=1}^{2} \omega_{i}^{2}}{\left(\sum_{i=1}^{2} \omega_{i}\right)^{2}}(\hat{u}-1)\right],
$$

where $\hat{u}=-2 u / \sum_{i=1}^{2} \omega_{i}$ and $u$ is a chemical potential for gauge or flavor symmetries. More precisely, denoting with $z$ the gauge variables, $E_{\mathrm{SUSY}}$ gets an additive contribution $\Psi\left(z+\Delta_{I}\right)$ from each chiral multiplet and $-\Psi(z)$ for each vector multiplet. One can pull out regularization factors from the matrix model only if they are independent of the gauge variables. The constraint (C.9) implies that $\sum_{I \in W_{a}} \hat{\Delta}_{I}=2$, where again $\hat{\Delta}_{I}=$ $-2 \Delta_{I} / \sum_{i=1}^{2} \omega_{i}$. Hence $\hat{\Delta}_{I}$ parameterizes a trial R-symmetry of the theory. One can see that, if all (pure and mixed) gauge anomalies cancel, $E_{\mathrm{SUSY}}$ is indeed independent of $z$ if the chemical potential satisfy (C.9) [13]. The final result for $E_{\mathrm{SUSY}}$ is then easily computed to be,

$$
E_{\mathrm{SUSY}}=i \pi \frac{\left(\sum_{i=1}^{2} \omega_{i}\right)^{3}}{24 \omega_{1} \omega_{2}}\left[\operatorname{Tr} R\left(\hat{\Delta}_{I}\right)^{3}-\frac{\sum_{i=1}^{2} \omega_{i}^{2}}{\left(\sum_{i=1}^{2} \omega_{i}\right)^{2}} \operatorname{Tr} R\left(\hat{\Delta}_{I}\right)\right] .
$$

Using (C.4) and $\omega_{i}=-i\left|b_{i}\right|$ we recover (C.3).

A similar quantity constructed from

$$
\tilde{\Psi}(u)=i \pi \frac{\left(\sum_{i=1}^{3} \omega_{i}\right)^{3}}{24 \omega_{1} \omega_{2} \omega_{3}}\left[(\hat{u}-1)^{3}-\frac{\sum_{i=1}^{3} \omega_{i}^{2}}{\left(\sum_{i=1}^{3} \omega_{i}\right)^{2}}(\hat{u}-1)\right],
$$

appears in the modular transformation of the integrand of the matrix model $[16] .{ }^{12}$ Here the angular momentum fugacities are written as $p=e^{-2 \pi i \omega_{1} / \omega_{3}}, q=e^{-2 \pi i \omega_{2} / \omega_{3}}$, the gauge fugacity as $e^{2 \pi u / \omega_{3}}$, and $\hat{u}=-2 u / \sum_{i=1}^{3} \omega_{i}$. In a theory with no gauge anomalies, the sum of $\tilde{\Psi}\left(z+\Delta_{I}\right)$ from each chiral multiplet and $-\tilde{\Psi}(z)$ for each vector multiplet is independent of the gauge variables $z$ and can be pulled out of the integral if the constraint

$$
\sum_{I \in W_{a}} \Delta_{I}+\sum_{i=1}^{3} \omega_{i}=0
$$

is fulfilled $[16,74]$. The sum then becomes

$$
\varphi=i \pi \frac{\left(\sum_{i=1}^{3} \omega_{i}\right)^{3}}{24 \omega_{1} \omega_{2} \omega_{3}}\left[\operatorname{Tr} R\left(\hat{\Delta}_{I}\right)^{3}-\frac{\sum_{i=1}^{3} \omega_{i}^{2}}{\left(\sum_{i=1}^{3} \omega_{i}\right)^{2}} \operatorname{Tr} R\left(\hat{\Delta}_{I}\right)\right] .
$$

This observation has been used in [16] to write the index as

$$
I\left(p, q, \Delta_{I}\right)=e^{\varphi} I^{\bmod }\left(\omega_{i}, \Delta_{I}\right),
$$

\footnotetext{
${ }^{12}$ This can be expressed in terms of Bernoulli polynomials as $\tilde{\Psi}(u)=\frac{\pi i}{3} B_{3,3}\left(u ; \omega_{i}\right)[16]$.
} 
where $I^{\text {mod }}$ is a modified matrix model depending on modified elliptic gamma functions. As noticed in [16], the supersymmetric Casimir energy can be extracted from $\varphi$ in the low temperature limit. Indeed, in the limit $\omega_{3}=1 / \beta \rightarrow 0, \varphi$ becomes exactly $\beta E_{\mathrm{SUSY}}$. It is interesting to notice that, for $\mathcal{N}=4 \mathrm{SYM}$, by setting one of the $\omega_{i}$ equal to $-1, \varphi$ reduces to our quantity (1.2) and (C.13) to the constraint (1.3). This is an observation to explore further. In particular, it would be interesting to understand the physical meaning of $I^{\text {mod }}$ and the decomposition (C.15).

Open Access. This article is distributed under the terms of the Creative Commons Attribution License (CC-BY 4.0), which permits any use, distribution and reproduction in any medium, provided the original author(s) and source are credited.

\section{References}

[1] J.B. Gutowski and H.S. Reall, Supersymmetric AdS 5 black holes, JHEP 02 (2004) 006 [hep-th/0401042] [INSPIRE].

[2] J.B. Gutowski and H.S. Reall, General supersymmetric AdS $S_{5}$ black holes, JHEP 04 (2004) 048 [hep-th/0401129] [INSPIRE].

[3] Z.W. Chong, M. Cvetič, H. Lü and C.N. Pope, Five-dimensional gauged supergravity black holes with independent rotation parameters, Phys. Rev. D 72 (2005) 041901 [hep-th/0505112] [INSPIRE].

[4] Z.W. Chong, M. Cvetič, H. Lü and C.N. Pope, General non-extremal rotating black holes in minimal five-dimensional gauged supergravity, Phys. Rev. Lett. 95 (2005) 161301 [hep-th/0506029] [INSPIRE].

[5] H.K. Kunduri, J. Lucietti and H.S. Reall, Supersymmetric multi-charge AdS $S_{5}$ black holes, JHEP 04 (2006) 036 [hep-th/0601156] [INSPIRE].

[6] J. Kinney, J.M. Maldacena, S. Minwalla and S. Raju, An Index for 4 dimensional super conformal theories, Commun. Math. Phys. 275 (2007) 209 [hep-th/0510251] [INSPIRE].

[7] L. Grant, P.A. Grassi, S. Kim and S. Minwalla, Comments on 1/16 BPS Quantum States and Classical Configurations, JHEP 05 (2008) 049 [arXiv:0803.4183] [INSPIRE].

[8] C.M. Chang and X. Yin, 1/16 BPS states in $\mathcal{N}=4$ super-Yang-Mills theory, Phys. Rev. D 88 (2013) 106005 [arXiv: 1305.6314] [INSPIRE].

[9] C. Romelsberger, Counting chiral primaries in $N=1, D=4$ superconformal field theories, Nucl. Phys. B 747 (2006) 329 [hep-th/0510060] [InSPIRE].

[10] B. Assel, D. Cassani and D. Martelli, Localization on Hopf surfaces, JHEP 08 (2014) 123 [arXiv: 1405.5144] [INSPIRE].

[11] J. Lorenzen and D. Martelli, Comments on the Casimir energy in supersymmetric field theories, JHEP 07 (2015) 001 [arXiv: 1412.7463] [INSPIRE].

[12] B. Assel, D. Cassani, L. Di Pietro, Z. Komargodski, J. Lorenzen and D. Martelli, The Casimir Energy in Curved Space and its Supersymmetric Counterpart, JHEP 07 (2015) 043 [arXiv: 1503.05537] [INSPIRE].

[13] N. Bobev, M. Bullimore and H.-C. Kim, Supersymmetric Casimir Energy and the Anomaly Polynomial, JHEP 09 (2015) 142 [arXiv:1507.08553] [INSPIRE]. 
[14] D. Martelli and J. Sparks, The character of the supersymmetric Casimir energy, JHEP 08 (2016) 117 [arXiv: 1512.02521] [INSPIRE].

[15] P. Benetti Genolini, D. Cassani, D. Martelli and J. Sparks, The holographic supersymmetric Casimir energy, Phys. Rev. D 95 (2017) 021902 [arXiv: 1606. 02724] [INSPIRE].

[16] F. Brünner, D. Regalado and V.P. Spiridonov, Supersymmetric Casimir energy and $\mathrm{SL}(3, \mathbb{Z})$ transformations, JHEP 07 (2017) 041 [arXiv:1611.03831] [INSPIRE].

[17] F. Benini, K. Hristov and A. Zaffaroni, Black hole microstates in AdS 4 from supersymmetric localization, JHEP 05 (2016) 054 [arXiv: 1511.04085] [INSPIRE].

[18] F. Benini, K. Hristov and A. Zaffaroni, Exact microstate counting for dyonic black holes in $A d S_{4}$, Phys. Lett. B 771 (2017) 462 [arXiv:1608.07294] [inSPIRE].

[19] F. Benini and A. Zaffaroni, A topologically twisted index for three-dimensional supersymmetric theories, JHEP 07 (2015) 127 [arXiv: 1504.03698] [INSPIRE].

[20] F. Benini and A. Zaffaroni, Supersymmetric partition functions on Riemann surfaces, arXiv: 1605.06120 [INSPIRE].

[21] A. Cabo-Bizet, Factorising the 3D Topologically Twisted Index, JHEP 04 (2017) 115 [arXiv: 1606.06341] [INSPIRE].

[22] C. Closset and H. Kim, Comments on twisted indices in 3d supersymmetric gauge theories, JHEP 08 (2016) 059 [arXiv: 1605.06531] [INSPIRE].

[23] C. Closset, H. Kim and B. Willett, Supersymmetric partition functions and the three-dimensional A-twist, JHEP 03 (2017) 074 [arXiv:1701.03171] [INSPIRE].

[24] S.M. Hosseini and A. Zaffaroni, Large- $N$ matrix models for $3 d \mathcal{N}=2$ theories: twisted index, free energy and black holes, JHEP 08 (2016) 064 [arXiv:1604.03122] [INSPIRE].

[25] S.M. Hosseini and N. Mekareeya, Large- $N$ topologically twisted index: necklace quivers, dualities and Sasaki-Einstein spaces, JHEP 08 (2016) 089 [arXiv:1604.03397] [INSPIRE].

[26] A. Cabo-Bizet, V.I. Giraldo-Rivera and L.A. Pando Zayas, Microstate Counting of $A d S_{4}$ Hyperbolic Black Hole Entropy via the Topologically Twisted Index, arXiv:1701.07893 [INSPIRE].

[27] S. Ferrara and R. Kallosh, Supersymmetry and attractors, Phys. Rev. D 54 (1996) 1514 [hep-th/9602136] [INSPIRE].

[28] S.L. Cacciatori and D. Klemm, Supersymmetric AdS 4 black holes and attractors, JHEP 01 (2010) 085 [arXiv:0911.4926] [INSPIRE].

[29] G. Dall'Agata and A. Gnecchi, Flow equations and attractors for black holes in $N=2 \mathrm{U}(1)$ gauged supergravity, JHEP 03 (2011) 037 [arXiv: 1012.3756] [INSPIRE].

[30] K. Hristov and S. Vandoren, Static supersymmetric black holes in AdS $S_{4}$ with spherical symmetry, JHEP 04 (2011) 047 [arXiv:1012.4314] [INSPIRE].

[31] S. Katmadas, Static BPS black holes in U(1) gauged supergravity, JHEP 09 (2014) 027 [arXiv: 1405.4901] [INSPIRE].

[32] N. Halmagyi, Static BPS black holes in AdS 4 with general dyonic charges, JHEP 03 (2015) 032 [arXiv: 1408.2831] [INSPIRE].

[33] D. Klemm, N. Petri and M. Rabbiosi, Symplectically invariant flow equations for $N=2$, $D=4$ gauged supergravity with hypermultiplets, JHEP 04 (2016) 008 [arXiv:1602.01334] [INSPIRE]. 
[34] A. Sen, Black hole entropy function and the attractor mechanism in higher derivative gravity, JHEP 09 (2005) 038 [hep-th/0506177] [INSPIRE].

[35] J.F. Morales and H. Samtleben, Entropy function and attractors for AdS black holes, JHEP 10 (2006) 074 [hep-th/0608044] [INSPIRE].

[36] M. Cvetič et al., Embedding AdS black holes in ten-dimensions and eleven-dimensions, Nucl. Phys. B 558 (1999) 96 [hep-th/9903214] [InSPIRE].

[37] J.P. Gauntlett, J.B. Gutowski and N.V. Suryanarayana, A Deformation of $A d S_{5} \times S^{5}$, Class. Quant. Grav. 21 (2004) 5021 [hep-th/0406188] [INSPIRE].

[38] H.K. Kunduri and J. Lucietti, Near-horizon geometries of supersymmetric AdS $S_{5}$ black holes, JHEP 12 (2007) 015 [arXiv: 0708.3695] [INSPIRE].

[39] S. Kim and K.-M. Lee, 1/16-BPS Black Holes and Giant Gravitons in the Ad $S_{5} \times S^{5}$ Space, JHEP 12 (2006) 077 [hep-th/0607085] [INSPIRE].

[40] K. Hristov, Dimensional reduction of BPS attractors in AdS gauged supergravities, JHEP 12 (2014) 066 [arXiv:1409.8504] [INSPIRE].

[41] D. Gaiotto, A. Strominger and X. Yin, New connections between $4-D$ and $5-D$ black holes, JHEP 02 (2006) 024 [hep-th/0503217] [INSPIRE].

[42] D. Gaiotto, A. Strominger and X. Yin, $5 D$ black rings and $4 D$ black holes, JHEP 02 (2006) 023 [hep-th/0504126] [INSPIRE].

[43] K. Behrndt, G. Lopes Cardoso and S. Mahapatra, Exploring the relation between $4-D$ and 5 - D BPS solutions, Nucl. Phys. B 732 (2006) 200 [hep-th/0506251] [INSPIRE].

[44] N. Banerjee, B. de Wit and S. Katmadas, The Off-Shell 4D/5D Connection, JHEP 03 (2012) 061 [arXiv:1112.5371] [INSPIRE].

[45] L. Andrianopoli, S. Ferrara and M.A. Lledó, Scherk-Schwarz reduction of D $=5$ special and quaternionic geometry, Class. Quant. Grav. 21 (2004) 4677 [hep-th/0405164] [INSPIRE].

[46] G. Lopes Cardoso, J.M. Oberreuter and J. Perz, Entropy function for rotating extremal black holes in very special geometry, JHEP 05 (2007) 025 [hep-th/0701176] [INSPIRE].

[47] O. Aharony, M. Berkooz, J. Louis and A. Micu, Non-Abelian structures in compactifications of M-theory on seven-manifolds with SU(3) structure, JHEP 09 (2008) 108 [arXiv:0806.1051] [INSPIRE].

[48] H. Looyestijn, E. Plauschinn and S. Vandoren, New potentials from Scherk-Schwarz reductions, JHEP 12 (2010) 016 [arXiv: 1008.4286] [INSPIRE].

[49] L. Andrianopoli, S. Ferrara and M.A. Lledó, No-scale $D=5$ supergravity from Scherk-Schwarz reduction of D =6 theories, JHEP 06 (2004) 018 [hep-th/0406018] [INSPIRE].

[50] L. Andrianopoli, M.A. Lledó and M. Trigiante, The Scherk-Schwarz mechanism as a flux compactification with internal torsion, JHEP 05 (2005) 051 [hep-th/0502083] [INSPIRE].

[51] K. Hristov and A. Rota, $6 d-5 d-4 d$ reduction of BPS attractors in flat gauged supergravities, Nucl. Phys. B 897 (2015) 213 [arXiv:1410.5386] [INSPIRE].

[52] D. Astefanesei, N. Banerjee and S. Dutta, Near horizon data and physical charges of extremal AdS black holes, Nucl. Phys. B 853 (2011) 63 [arXiv:1104.4121] [INSPIRE].

[53] E. Witten, Mirror manifolds and topological field theory, hep-th/9112056 [INSPIRE].

[54] I. Papadimitriou, Supercurrent anomalies in 4d SCFTs, JHEP 07 (2017) 038 [arXiv: 1703. 04299] [INSPIRE]. 
[55] O.S. An, Anomaly-corrected supersymmetry algebra and supersymmetric holographic renormalization, arXiv:1703.09607 [INSPIRE].

[56] A. Arabi Ardehali, J.T. Liu and P. Szepietowski, High-Temperature Expansion of Supersymmetric Partition Functions, JHEP 07 (2015) 113 [arXiv:1502.07737] [INSPIRE].

[57] A. Arabi Ardehali, High-temperature asymptotics of supersymmetric partition functions, JHEP 07 (2016) 025 [arXiv: 1512.03376] [INSPIRE].

[58] E. Shaghoulian, Modular invariance on $S^{1} \times S^{3}$ and circle fibrations, arXiv:1612.05257 [INSPIRE].

[59] L. Di Pietro and M. Honda, Cardy Formula for 4d SUSY Theories and Localization, JHEP 04 (2017) 055 [arXiv: 1611.00380] [INSPIRE].

[60] F. Nieri and S. Pasquetti, Factorisation and holomorphic blocks in 4d, JHEP 11 (2015) 155 [arXiv: 1507.00261] [INSPIRE].

[61] S. Yankielowicz and Y. Zhou, Supersymmetric Rényi entropy and Anomalies in $6 d(1,0)$ SCFTs, JHEP 04 (2017) 128 [arXiv: 1702.03518] [INSPIRE].

[62] S. Ferrara and R. Kallosh, Universality of supersymmetric attractors, Phys. Rev. D 54 (1996) 1525 [hep-th/9603090] [INSPIRE].

[63] A.H. Chamseddine, S. Ferrara, G.W. Gibbons and R. Kallosh, Enhancement of supersymmetry near $5-D$ black hole horizon, Phys. Rev. D 55 (1997) 3647 [hep-th/9610155] [INSPIRE].

[64] R. Kallosh, A. Rajaraman and W.K. Wong, Supersymmetric rotating black holes and attractors, Phys. Rev. D 55 (1997) 3246 [hep-th/9611094] [INSPIRE].

[65] S. Barisch-Dick, G. Lopes Cardoso, M. Haack and S. Nampuri, Extremal black brane solutions in five-dimensional gauged supergravity, JHEP 02 (2013) 103 [arXiv:1211.0832] [INSPIRE].

[66] D. Klemm, N. Petri and M. Rabbiosi, Black string first order flow in $N=2, d=5$ abelian gauged supergravity, JHEP 01 (2017) 106 [arXiv:1610.07367] [INSPIRE].

[67] A. Amariti and C. Toldo, Betti multiplets, flows across dimensions and c-extremization, JHEP 07 (2017) 040 [arXiv:1610.08858] [INSPIRE].

[68] S.M. Hosseini, A. Nedelin and A. Zaffaroni, The Cardy limit of the topologically twisted index and black strings in $A d S_{5}$, JHEP 04 (2017) 014 [arXiv: 1611.09374] [INSPIRE].

[69] F. Benini and N. Bobev, Exact two-dimensional superconformal R-symmetry and c-extremization, Phys. Rev. Lett. 110 (2013) 061601 [arXiv:1211.4030] [INSPIRE].

[70] F. Benini and N. Bobev, Two-dimensional SCFTs from wrapped branes and c-extremization, JHEP 06 (2013) 005 [arXiv:1302.4451] [INSPIRE].

[71] L. Andrianopoli et al., $N=2$ supergravity and $N=2$ super Yang-Mills theory on general scalar manifolds: Symplectic covariance, gaugings and the momentum map, J. Geom. Phys. 23 (1997) 111 [hep-th/9605032] [INSPIRE].

[72] J. Louis and A. Micu, Type 2 theories compactified on Calabi-Yau threefolds in the presence of background fluxes, Nucl. Phys. B 635 (2002) 395 [hep-th/0202168] [INSPIRE].

[73] N. Halmagyi, M. Petrini and A. Zaffaroni, BPS black holes in AdS 4 from M-theory, JHEP 08 (2013) 124 [arXiv:1305.0730] [INSPIRE].

[74] V.P. Spiridonov and G.S. Vartanov, Elliptic hypergeometric integrals and 't Hooft anomaly matching conditions, JHEP 06 (2012) 016 [arXiv: 1203.5677] [INSPIRE]. 